\title{
Percepción de las emociones del alumnado universitario del grado de Educación Infantil al implementar estrategias y actividades online en el aprendizaje en didáctica de las ciencias
}

\author{
Juan-Francisco Álvarez-Herrero*
}

Universidad de Alicante

\author{
PALABRAS CLAVE \\ Formación de docentes \\ Didáctica de la Ciencia \\ E-learning \\ Estrategias de aprendizaje
}

\begin{abstract}
RESUMEN
Con el confinamiento provocado por la pandemia de la COVID-19, el mundo educativo se vio abocado a una modalidad de enseñanza online y al uso continuado de las tecnologías digitales. En asignaturas como las de ciencias, más manipulativas e interactivas, ello supuso un verdadero reto. En esta experiencia recogemos las percepciones de 46 estudiantes universitarios sobre si el uso de unas determinadas estrategias y actividades les condujo a una mayor motivación hacía las ciencias. Para ello, tras poner en práctica dichas estrategias, se les pasó un pequeño cuestionario de cuyas respuestas se desprende que el alumnado valoró muy positivamente dichas experiencias y perciben una mejora sustancial de sus emociones y sensaciones hacía las ciencias. Si bien las actividades mejor valoradas son aquellas que requieren de una mayor interacción y participación por su parte, también ven en esta mayor implicación un problema. Se discute y se cree necesario un plan de formación docente que vaya más allá del conocimiento y uso de tecnología digital y que forme en la elección, creación, desarrollo e implementación de las estrategias y actividades adecuadas que permitan una mejora no sólo en la motivación sino también en el aprendizaje del alumnado.
\end{abstract}

Perception of emotions of university students of the degree of Early Childhood Education when implementing strategies and activities to the online learning of science education

\begin{abstract}
With the confinement caused by the COVID-19 pandemic, the educational world was forced to an online teaching modality and the continued use of digital technologies. In subjects such as science, which were more manipulative and interactive, this was a real challenge. In this experience we collected the perceptions of 46 university students on whether the use of certain strategies and activities led them to greater motivation towards science. To do this, after putting these strategies into practice, they were given a short questionnaire from whose responses it is clear that the students valued these experiences very positively and perceive a substantial improvement in their emotions and feelings towards science. Although the most highly valued activities are those that require greater interaction and participation on their part, they also see this greater involvement
\end{abstract}

\section{Universidad de Alicante}

Autor de correspondencia: * Juan-Francisco Álvarez-Herrero; juanfran.alvarez@ua.es

Recibido: 15/05/2021 - Aceptado: 15/09/2021

Revista de Formación del Profesorado e Investigación Educativa

Facultad de Formación del Profesorado y Educación

Universidad de Oviedo

Enero - Diciembre 2021

ISSN: $2340-4728$

(c) (1) () $\odot$ Esta obra está bajo una licencia internacional Creative Commons

Esta obra está bajo una licencia internacional
Atribución-NoComercial-SinDerivadas 4.0. 
as a problem. A teacher training plan that goes beyond the knowledge and use of digital technology is discussed and is believed to be necessary and that forms in the choice, creation, development and implementation of appropriate strategies and activities that allow an improvement not only in motivation but also in student learning.

\section{Introducción}

La pandemia del coronavirus en el mundo educativo ha hecho resurgir las tecnologías digitales. El tener que cambiar de forma repentina de una modalidad presencial de enseñanza a otra online, puso en jaque a instituciones educativas, al profesorado y al alumnado. Tanto los recursos y las estrategias, como lo preparados y competentes que fuesen digitalmente docentes y discentes, se vieron comprometidos de la noche a la mañana (König et al., 2020). Así, la situación durante el confinamiento se salvó como mejor se pudo con la implicación de todas las partes (Hernández-Ortega y Álvarez-Herrero, 2021). Sin embargo, ello no estuvo exento de problemas y críticas. Son varios los estudios que han analizado los problemas que se presentaron (Adnan y Anwar, 2020; Lorente et al., 2020), así como las percepciones de docentes y discentes sobre las dificultades encontradas o las ventajas para el aprendizaje de esta modalidad de enseñanza (Lozano-Díaz et al., 2020; Rodicio-García et al., 2020; Shehzadi et al., 2020). Entre los problemas que más se destacan en estas investigaciones, cabe destacar entre otros: la falta de dispositivos, deficiente o nula conectividad a internet, la falta de competencia digital tanto de docentes como de discentes, o el tener que compartir los dispositivos con otros miembros de la familia (Rahiem, 2020). También, aunque se haya hablado menos del mismo, la ansiedad, el estrés y los trastornos mentales que este uso continuado de la tecnología supuso, es un problema a tener muy en cuenta y que obliga a buscar soluciones en un futuro en pro de un uso equilibrado de la tecnología (Lee et al., 2021; Wind et al., 2020). Por otro lado, el uso de las tecnologías digitales siempre resulta muy motivador para el alumnado. Se trata de un aliciente que bien aprovechado, permite tanto al profesorado como al alumnado obtener un valor añadido al aprendizaje. Además, en la situación que nos ocupa, el no tener que desplazarse hasta un lugar, el poder ser flexible en el tiempo, la inmediatez, la gran cantidad de recursos disponibles, así como la facilidad de obtención de información y comunicación, hacen de las tecnologías digitales, las perfectas compañeras en esta modalidad de enseñanza online.

Uno de los problemas más importantes a los que se enfrentaron los y las docentes, fue el llevar a la modalidad online, no presencial, los contenidos y aprendizajes que tan trabajados tienen en la modalidad presencial. En muchos casos, teniendo todo tipo de recursos a su disposición, el proceso de transformación ha sido una simple adaptación de los mismos a esta nueva modalidad (García-Planas y Taberna, 2020; Menéndez, 2020; Van Vaerenbergh, 2020). Sin embargo, esto no garantiza un aprendizaje eficaz ni es la mejor opción en todas las ocasiones y materias. Así, por ejemplo, en las materias de ciencias resulta bastante complicado, pues estas exigen una mayor interacción y participación del alumnado, que a la postre debe ser quien investigue y haga Ciencia por sí mismo para conseguir construir conocimiento y aprender de una forma significativa. En un aprendizaje presencial resulta bastante fácil realizar todo este tipo de prácticas y acciones, pero un aprendizaje no presencial requiere otro tipo de estrategias y recursos para poder llevar a cabo el aprendizaje de las ciencias con garantías (Álvarez-Herrero y Fernández-Herrero, 2020).

Es decir, no vale con transmitir al alumnado contenidos en diferentes formatos (videoconferencias, videos grabados, pdfs, etc.) y exigirle la realización en casa de tareas y actividades puntuales, puesto que con ello no estamos para nada fomentando el pensamiento crítico o el desarrollo de la competencia científica. De ahí que no sea válida una simple adaptación de los materiales y contenidos teóricos y prácticos de las asignaturas de ciencias a una modalidad de enseñanza no presencial y por tanto se requiera una evolución que contemple otro tipo de estrategias y recursos para la modalidad online (Torralba-Burrial, 2020). Dado que esta vez las tecnologías digitales y algunas de las estrategias y recursos utilizados durante el confinamiento, parece que han venido para quedarse (Ali, 2020), es muy posible que en unos años tanto docentes como discentes se habitúen al uso de estos. Con el paso del tiempo, esa exigencia de un mayor esfuerzo y trabajo por parte del profesorado en el diseño, creación y ejecución de estas estrategias y recursos, será mucho menor, propiciando también su consolidación (Paudel, 2021). Si bien, los contenidos y recursos seguirán mayoritariamente siendo aportados por las editoriales, muy probablemente cada vez también será mayor el número de docentes que prefieran utilizar estrategias y recursos propios, pues quién mejor que ellos y ellas para conocer las necesidades y la forma de aprender de su alumnado, y a su vez conseguir de esta manera una enseñanza más personalizada (Adedoyin y Soykan, 2020; Rueda-Gómez, 2020).

Si bien hay materias que no requieren tanto de un razonamiento, o de poner en juego el pensamiento crítico, la investigación y la comprobación, cuando hablamos de obtener un buen aprendizaje de las ciencias y su didáctica, todas estas capacidades deben ser puestas en juego y se hace necesario un buen desarrollo de la competencia científica, además de otras muchas competencias más (Álvarez-Herrero y Valls-Bautista, 2019). De ahí, que sea tan importante en esta modalidad online, que las estrategias y actividades que se propongan vayan en esta dirección (Álvarez-Herrero, 2020), que sean capaces de lograr que sea el alumnado el protagonista de su aprendizaje, interaccionando y actuando mucho más allá de los contenidos teóricos, poniendo en práctica todo aquello que va descubriendo para una correcta construcción de conocimiento.

El alumnado universitario de los grados de educación puede estar más familiarizado con metodologías, estrategias y recursos pedagógicos, y en general, es también este alumnado quien, por su edad, grado de madurez e independencia, puede estar mejor preparado para afrontar con mayores garantías una enseñanza online. Sin embargo, tanto el alumnado como el profesorado del grado de magisterio en Educación Infantil, están igualmente condicionados por su grado o nivel de competencia digital que posean (Cabero-Almenara y Llorente-Cejudo, 2020). Así, si dicho alumnado y profesorado cuentan con un elevado grado de dicha competencia, tienen mayores posibilidades de aprender con mayor motivación unos, y de saber guiar y acompañar en dicho proceso otros (Sandoval, 2020). No bastará pues, el evolucionar a una modalidad de aprendizaje no presencial con otro tipo de estrategias y recursos más adecuados, sino que también es necesaria una óptima competencia digital tanto de alumnado como de profesorado (Cóndor-Herrera, 2020).

\section{Objetivos}

En esta investigación quisimos conocer cuáles eran las percepciones del alumnado universitario del grado de magisterio en Educación Infantil en el área de didáctica de las ciencias experimentales, respecto a si se produce una mejora de sus emociones y 
sensaciones hacia las ciencias tras el uso de diferentes estrategias y actividades en la clase online y mediadas con tecnología. Así mismo, quisimos conocer cuáles eran las principales ventajas e inconvenientes que detectaron en este tipo de estrategias y actividades, frente a las habitualmente utilizadas en las clases presenciales.

\section{Metodología}

\section{Muestra}

La investigación que aquí nos ocupa analiza la experiencia llevada a cabo con un grupo de estudiantes del grado de Maestro en Educación Infantil de la Universidad de Alicante en su campus de Alcoi. Se trata de un grupo que en dicho momento se encontraba cursando la asignatura de Didáctica del Conocimiento del Medio Natural en horario vespertino y en la lengua autóctona de la comunidad valenciana, el valenciano. Es un grupo bastante cohesionado, donde todos los y las estudiantes se conocen entre sí, y cuentan con un grupo de WhatsApp con el que se mantiene la comunicación y la información de todo lo que les atañe. Su procedencia es variada y así hay estudiantes que proceden de Alcoi, pero también de otras muchas ciudades y pueblos de las provincias de Alicante y Valencia. La asignatura está enfocada desde una metodología activa basada en la indagación y con una estructura de planteamiento a modo de secuencias problematizadas donde el alumnado va descubriendo, indagando y tratando de dar respuesta a las preguntas que el docente plantea, quien a su vez les va guiando en su aprendizaje y construcción de su propio conocimiento de ciencias. En numerosas ocasiones este enfoque obliga a la realización de prácticas experimentales con las que contrastar y comprobar lo que se va descubriendo e investigando.

De los 49 estudiantes matriculados en la asignatura, tan sólo 46 participaron de forma activa durante el periodo de actividad de la asignatura y son los que aquí se recogen. De estos 46 estudiantes, 39 son mujeres $(84,8 \%)$ y 7 son hombres $(15,2 \%)$, con edades comprendidas entre los 20 y los 37 años y una edad media establecida en 22,7 años.

\section{Instrumento de recogida de información}

En el último día de clase de la asignatura y tras haber recogido y así expresado, el consentimiento de todo el alumnado a participar en la recogida de datos, se les pasó un cuestionario elaborado ad hoc para la ocasión, similar al utilizado en otras investigaciones (Pozo-Sánchez et al., 2021; Villalustre et al., 2019). Dicho cuestionario se confeccionó con la herramienta de Google Forms y se dispuso en línea para su cumplimentación. Además de las preguntas sobre su sexo y edad, el cuestionario incluía únicamente 4 sencillas preguntas. Una de escala tipo Likert, del 1 (Nada) al 5 (Mucho) en la que se les preguntaba sobre su percepción en cuanto a una mejora o no en su aprendizaje tras una enseñanza online. Una segunda pregunta les pedía que valorasen el grado de aceptación de 10 de las estrategias y actividades realizadas en la asignatura, también sobre esa misma escala del 1 al 5. Y, por último, dos preguntas abiertas en las que manifestasen por un lado las ventajas y virtudes de trabajar en una modalidad online mediante este tipo de estrategias y actividades, y, por otro lado, los problemas e inconvenientes. Los enunciados de estas 4 preguntas se pueden ver en la tabla del Anexo 1. Se buscó que el cuestionario fuese corto y sencillo de responder, para así permitir respuestas lo más ajustadas a la percepción real del alumnado sin ningún tipo de condicionante que pudiese falsear los resultados.

\section{Procedimiento}

La situación sanitaria derivada de la pandemia de la COVID-19 obligó a suspender las clases presenciales y estas pasaron a realizarse de forma online. La asignatura se vio directamente afectada y ello hizo que se aprovechase para transformar o mejor, evolucionar que no adaptar, las estrategias y los recursos a utilizar para seguir manteniendo ese componente de indagación, interacción y participación-acción propio de la asignatura. Todo el alumnado del grupo trabajó de forma no presencial la asignatura, siguiendo las clases mediante videoconferencias y realizando las actividades marcadas por el profesor de la asignatura. A continuación, se describen las actividades y estrategias utilizadas en dichas clases online:

a) Videoconferencias o videoclases expositivas por parte del profesor, realizadas a través de Google meet y donde el profesor transmitía contenidos durante unos tiempos cortos de entre 20 y 30 minutos. El alumnado asistía a dichas videoconferencias, pero no tenían la obligación de tener que encender ni cámara ni micro. Sí podían participar con intervenciones a modo de preguntas o comentarios, pero estos eran muy raros y ocasionales.

b) Videoclases interactivas con preguntas síncronas con Mentimeter. A la vez que se iba trabajando en los contenidos del área, el profesor iba lanzando preguntas indagatorias al alumnado a través de aplicaciones como Mentimeter y Nearpood que permiten cuestionar y pedir la interacción del alumnado de forma inmediata. Hasta no estar resuelta una cuestión, no se pasaba a la siguiente.

c) Visualización de forma individual de videos de YouTube con preguntas en EdPuzzle. El docente proporcionaba enlaces a videos de YouTube, en los que en muchas ocasiones estos habían sido preparados previamente con la aplicación EdPuzzle con la introducción de preguntas que se insertaban en cualquier momento del video y que permitían comprobar al docente si el alumnado iba comprendiendo lo que estaba viendo en dichos videos. El alumnado podía visualizar las veces que quisiese el video, rebobinarlo, avanzarlo, pararlo, etc., y el docente recibía un informe personalizado de cada estudiante, pudiendo de esta manera conocer el avance en el aprendizaje de cada uno/a y a su vez convertir dicha experiencia en un aprendizaje más personalizado.

d) Trabajo en grupo en salas alternativas de Google meet. En ocasiones se buscó la participación e interacción entre iguales. De ahí que a partir de una sala de meet conjunta de todo el grupo clase, se derivaban otras salas de meet, una para cada grupo de 4 ó 5 estudiantes, que durante tiempos programados trabajaban en estos equipos las actividades propuestas por el docente. Al haber sido el propio docente quien había creado las salas, este podía pasarse por las diferentes salas, supervisando, guiando, y contestando a los requerimientos y preguntas que los grupos tuviesen a bien hacerle.

e) Formularios en línea con Google Forms. Para recabar el trabajo realizado por el alumnado en actividades y prácticas, en ocasiones se le planteó al alumnado la realización de estas actividades en formularios con los enunciados de las mismas y que debían de entregar en un tiempo determinado. Posteriormente, bien se realizaba una corrección conjunta de dichas actividades en forma de videoconferencia, bien se les facilitaba un documento con las respuestas a dichas actividades para que cada estudiante pudiese corregirse y supervisarse lo que había hecho.

f) Cuestionarios en línea con Kahoot y Quizizz. La inmediatez y rapidez de estos cuestionarios, se utilizó para constatar si se iban asimilando e interiorizando los contenidos que se iban trabajando. Se trató de quitar peso a la competitividad que este tipo de aplicaciones fomentan, aunque esta en ocasiones también fue buena para permitir que incentivase el aprendizaje de aquellos y aquellas estudiantes que iban un poco más rezagados. 
g) Realización de glosarios y repositorios de forma colaborativa. Se propuso en dos ocasiones la realización de un glosario de términos científicos y un repositorio de recursos sobre los contenidos científicos trabajados, donde de forma conjunta, todo estudiante debía contribuir con un par de términos o recursos, sin que estos se repitiesen entre todos los elaborados por toda la clase. Ello permitió la realización de estos trabajos de una forma colaborativa, proporcionando al alumnado un material bastante enriquecedor.

h) Prácticas de ciencias experimentales mediadas con tecnología (la caja negra). Algunas prácticas se mandaron realizar de forma individual al alumnado en sus casas y con materiales y utensilios sencillos que pudiesen tener $\mathrm{o}$ fuesen fáciles de obtener. Otras fueron lanzadas a través de laboratorios virtuales presentes en Internet. Pero particularmente, fue bastante exitosa la realización de una de las prácticas siempre presentes en esta asignatura como es la de: La caja negra. En ella el alumnado, en grupos, debe ser capaz de ir constatando y diseñando una experiencia que le permita conocer el interior de una pequeña caja que no puede abrir hasta el final de la práctica. Los pasos en los que el docente guía al alumnado en el desarrollo de esta práctica se corresponden con los pasos o etapas del método científico. Aunque al principio de la práctica, el alumnado no ha de disponer de la misma para hacer sus primeras hipótesis, sí cuando se pasa a la experimentación era necesario que de alguna manera el alumnado pudiese disponer de la caja. Esto se sustituyó por numerosas combinaciones de videos que el docente preparó, con todas las posibilidades de experimentación que se podían prever. Si bien el alumnado no pudo sentir las cajas, tocarlas, escucharlas, etc. de primera mano, sí que estos videos fueron un excelente sustituto de la presencialidad.

i) Indagación guiada (WebQuests). Se contó con un par de actividades enfocadas a modo de WebQuests, donde el alumnado tiene que buscar información para mediante la indagación llegar al conocimiento de determinados conceptos. En dichas WebQuests se le proporcionó al alumnado enlaces y pistas sobre dónde encontrar la información que necesitaban para ir contestando a las preguntas que se les planteaban.

j) Breakout edu (recapitulación de los contenidos trabajados). Como actividad de conclusión, en el último día de la asignatura, se planteó al alumnado la realización de un breakout edu. Se trata de una especie de yincana de pruebas con un tiempo delimitado y que se realizó en Internet haciendo uso de los recursos y herramientas que esta ofrece. En ella, el alumnado tuvo que ir resolviendo dichas pruebas/acertijos/retos para llegar a una meta final. Todas las pruebas tenían relación con los contenidos trabajados en la asignatura. Para pasar de una prueba a la siguiente, la primera debía ser resuelta y así sucesivamente hasta la última.

\section{Análisis de datos}

Los resultados se analizaron con el programa estadístico IBM SPSS Statistics v. 25, realizando una estadística descriptiva y analizando valores medios con sus desviaciones típicas y varianzas; y con hojas de Microsoft Excel, en las que se codificaron las respuestas y se contabilizaron frecuencias.

\section{Resultados}

Ante la primera pregunta, en la que se cuestiona al alumnado sobre su percepción acerca de si las estrategias y actividades llevadas a cabo con esta modalidad no presencial de la enseñanza-aprendizaje de la didáctica de las ciencias, les hacía percibir una mejora en su aprendizaje o no; se obtuvieron los resultados que se pueden observar en la Tabla 2.

Tabla 2. Valoración media sobre la percepción de una mejora en el aprendizaje de la didáctica de las ciencias en una modalidad online con otras estrategias y actividades.

\begin{tabular}{|l|c|c|c|c|}
\cline { 2 - 5 } \multicolumn{1}{c|}{} & Frecuencia & Media & $\begin{array}{c}\text { Desviación } \\
\text { típica }\end{array}$ & Varianza \\
\hline Pregunta 1 & 46 & 4,72 & 0,584 & 0,341 \\
\hline
\end{tabular}

La autopercepción de mejora de la motivación hacía las ciencias mediante este tipo de estrategias y actividades online por parte del estudiante, obtiene un valor medio excelente $(9,44$ sobre 10$)$. Esto nos hace presuponer que el alumnado está bastante contento con las sensaciones que estas estrategias y actividades les reportan y considera que mejoran sustancialmente su motivación hacia las ciencias frente a la práctica habitual de aprendizaje presencial.

En la segunda pregunta se trataba de ver la aceptación de las diferentes estrategias y actividades realizadas, para de esta manera comprobar si algunas de ellas prevalecen frente a otras. Los resultados obtenidos los podemos ver desglosados en la figura 1.

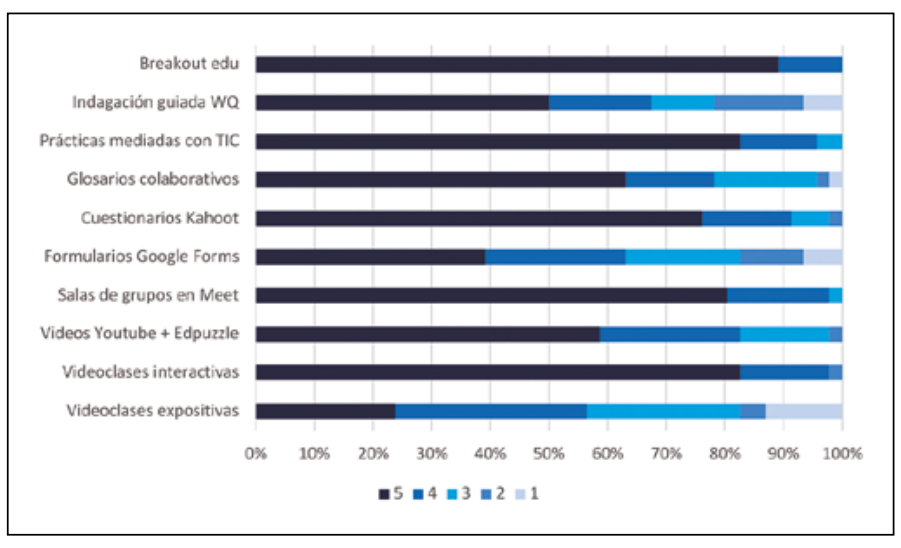

Fuente: Elaboración Propia

Figura 1. Valoración de las diferentes estrategias y actividades utilizadas

Tal y como se puede apreciar en la Figura 1, aquellas actividades que más se parecen a las actividades tradicionales presenciales: clases expositivas y realización de tareas o actividades a entregar, aquí en forma de videoclases expositivas y formularios de Google Forms son las que peor valoración obtienen. A estas dos podemos ver cómo se le une una tercera, la indagación guiada (WebQuests), que son las tres cuyos porcentajes de la máxima valoración (5) están en el 50 \% o por debajo de este. En cambio, podemos ver cómo las actividades mejor valoradas son: los breakout $e d u$, las prácticas mediadas con TIC, las videoclases interactivas, las salas de Meet para el trabajo en grupo y los cuestionarios interactivos con Kahoot o Quizizz. Se trata de actividades donde la participación, interacción y la colaboración están muy presentes y el alumnado valora muy positivamente este tipo de acciones al igual que ocurre en la investigación de Reinoso et al. (2021). En un término medio, sin llegar a estar mal valoradas, se encuentran la realización de glosarios y repositorios de forma colaborativa y el visionado de videos que se complementan con preguntas realizadas con la aplicación Edpuzzle, a modo de videocuestionarios.

Se detecta que el alumnado de mayor edad (24-37 años) es quien otorga las puntuaciones más elevadas a aquellas activida- 
des que se parecen más a las actividades tradicionales presenciales, y el alumnado más joven (20-23 años) el que puntúa mejor las actividades basadas en metodologías activas y que son más innovadoras. No podemos establecer una clara diferenciación de los resultados atendiendo a la variable sexo, pues como ya hemos comentado, la muestra está formada mayoritariamente por mujeres que es el colectivo que preferentemente escoge la realización de este grado de Magisterio en Educación Infantil.

Para analizar las ventajas y los inconvenientes destacados por el alumnado, dado el carácter abierto de estas dos últimas preguntas del cuestionario, se han contabilizado frecuencias de aquellos aspectos más citados. Las ventajas más comentadas se pueden apreciar en la Figura 2 y los inconvenientes en la Figura 3.

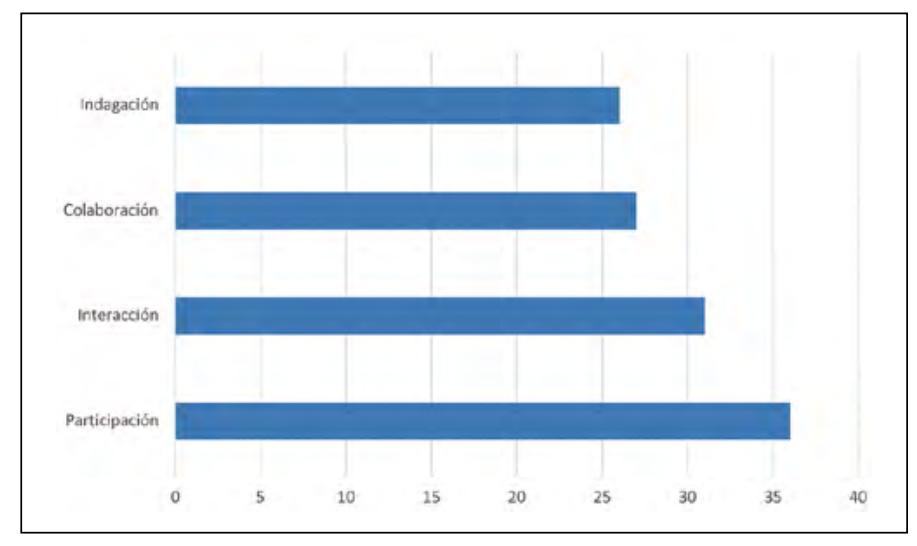

Fuente: Elaboración Propia

Figura 2. Ventajas destacadas por el alumnado

Como se puede apreciar en la Figura 2, la posibilidad de participar, interactuar, colaborar con otros e indagar/investigar son las ventajas que el alumnado aprecia con mayor valoración, dado que un elevado número de estudiantes las citan en sus respuestas.

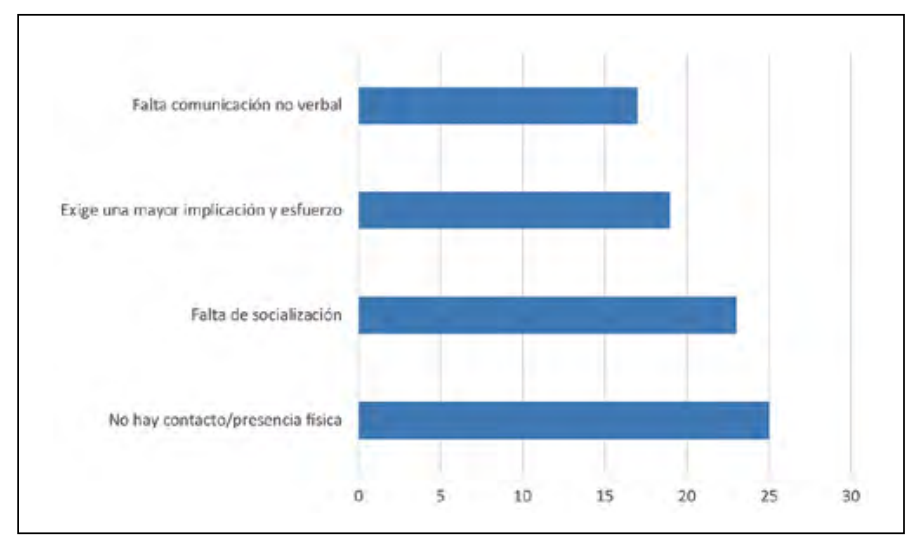

Fuente: Elaboración Propia

Figura 3. Inconvenientes destacados por el alumnado

En cambio, tal y como se aprecia en la Figura 3, los inconvenientes más nombrados por el alumnado inciden en echar en falta el contacto y la presencia física, la socialización y la comunicación no verbal, así como el que este tipo de estrategias y actividades online requieren una mayor implicación y esfuerzo por su parte.

Así pues, hemos visto como una mayor interacción y participación, son dos de los aspectos más valorados como ventajas de esta práctica, mientras que esa mayor implicación que exige este tipo de actividades es también vista como un inconveniente.

\section{Conclusiones y discusión}

El alumnado universitario del grado de Maestro en Educación Infantil en la asignatura de Didáctica del Conocimiento del Medio Natural, expuesto a un conjunto de estrategias y actividades en la modalidad online, valoró muy positivamente la eficacia que estas tienen según su percepción en la mejora de su motivación y sensaciones hacía las ciencias. Así se desprende de los resultados que hemos obtenido y eso nos permite afirmar que los estudiantes se han sentido cómodos con esta metodología de trabajo y que no sólo han valorado positivamente la experiencia por el uso de las tecnologías digitales, sino también por la metodología a la que ha evolucionado las clases al pasar de presenciales a online. Esto coincide plenamente con las investigaciones de Vélez-Loor et al. (2020) y de Tirado et al. (2021).

De estas estrategias y actividades, las mejor valoradas han sido aquellas en las que se requiere una participación e interacción continua del alumnado. Así, el breakout edu, las prácticas mediadas con tecnología, las salas colaborativas de trabajo en grupo y las videoclases interactivas, son las mejor valoradas. En el otro lado tenemos aquellas actividades que más se parecen a la enseñanza tradicional o que simplemente han sido adaptaciones de la enseñanza presencial a la online, como: las videoclases expositivas y el rellenar formularios de Google Forms con actividades en las que tanto el papel del alumnado y del profesorado han cambiado por esta modalidad no presencial de enseñanza. Esta interacción, continua participación y protagonismo del alumnado es también lo que más positivamente han valorado a la hora de hablar de las ventajas que esta experiencia les ha proporcionado. Sin embargo, el hecho de no estar acostumbrados a este tipo de dinámicas y estrategias, hace que también esta mayor exigencia de continua implicación y esfuerzo, sea visto como un problema o inconveniente, aun a pesar de que reconocen que mejora su motivación e interés por aprender ciencias. No se trata de ninguna contradicción, sino de que el alumnado se hace consciente de que en los procesos de enseñanza-aprendizaje a los que estaba acostumbrado, su protagonismo y participación era menor, que lo que exige esta modalidad online. En ella, las estrategias y actividades buscan una mayor interacción y participación activa del alumnado en la construcción de su propio aprendizaje, donde es él quien debe realizarse las preguntas y tratar de darles respuestas (Lorduy y Naranjo, 2020). Y dado que no está habituado a ello, exige un mayor trabajo y esfuerzo por su parte.

El uso de la tecnología en esta modalidad no presencial de la enseñanza-aprendizaje, obliga a que, pensando en un futuro inmediato, se establezcan planes de formación por las autoridades educativas competentes y por las diferentes instituciones. Estos planes deben ir dirigidos tanto al profesorado como al alumnado, en desarrollar su competencia digital, y en el caso de los docentes, ir más allá y proporcionarles formación en el tipo de estrategias, actividades y recursos que pueden utilizar en sus clases, bien sean en una modalidad presencial, semipresencial u online. Por todo ello, sea en la modalidad de enseñanza que sea, se hace necesaria una formación del profesorado que vaya más allá del conocimiento y uso de herramientas y recursos digitales, y que incida en argumentos pedagógicos que le permitan saber crear, desarrollar y aplicar las estrategias y actividades adecuadas para la mejora del aprendizaje de su alumnado. Coincidimos con otras investigaciones que también recomiendan una formación en tecnologías digitales centradas en la correcta elección, desarrollo y aplicación de estas estrategias y actividades (Kang, 2021; Naresh, 2020).

\section{Agradecimientos}

El trabajo no ha recibido financiación para su desarrollo. 


\section{Referencias}

Adedoyin, O. B., y Soykan, E. (2020). Covid-19 pandemic and online learning: the challenges and opportunities. Interactive Learning Environments, 1-13. https://doi.org/10.1080/10494820 2020.1813180

Adnan, M., y Anwar, K. (2020). Online Learning amid the COVID-19 Pandemic: Students' Perspectives. Online Submission, 2(1), 45-51. http://www.doi.org/10.33902/JPSP.202020261309

Ali, W. (2020). Online and remote learning in higher education institutes: A necessity in light of COVID-19 pandemic. Higher Education Studies, 10(3), 16-25. https://doi.org/10.5539/hes. v10n3p16

Álvarez-Herrero, J. F. (2020). Aprendizaje de las ciencias por indagación, en modalidad online, con alumnado de secundaria y alumnado universitario y en tiempos de la COVID-19. Dos realidades distintas, un nexo común y un argumento diferenciador. Educación Química, 31(5), 60-65. http://dx.doi. org/10.22201/fq.18708404e.2020.5.77091

Álvarez-Herrero, J. F. y Fernández-Herrero, J. (2020). Aprendizaje online versus presencial. Percepciones del alumnado del grado de Magisterio en Educación Infantil. En R. Roig-Vila (Ed.), La docencia en la Enseñanza Superior. Nuevas aportaciones desde la investigación e innovación educativas (pp. 5-12). Octaedro.

Álvarez Herrero, J. F., y Valls Bautista, C. (2019). Didáctica de las ciencias, ¿de dónde venimos y hacia dónde vamos? Revista de Ciències de l'Educació, 1(2), 5-19. https://doi.org/10.17345/ ute.2019.2.2704

Cabero-Almenara, J., y Llorente-Cejudo, C. (2020). Covid-19: transformación radical de la digitalización en las instituciones universitarias. Campus Virtuales, 9(2), 25-34.

Cóndor-Herrera, O. M. A. R. (2020). Educar en tiempos de COVID-19. CienciAmérica, 9(2), 31-37. http://dx.doi.org/10.33210/ ca.v9i2.281

García-Planas, M. I., y Taberna Torres, J. (2020). The transition from the classroom to non-classroom teaching at the UPC during the COVID-19 pandemic. IJERI: International Journal of Educational Research and Innovation, 15, 177-187. https://doi. org/10.46661/ijeri.5015

Hernández-Ortega, J., y Álvarez-Herrero, J.F. (2021). Gestión educativa del confinamiento por COVID-19: percepción del docente en España. Revista Española de Educación Comparada, 38, 129-150. https://doi.org/10.5944/reec.38.2021.29017

Kang, B. (2021). How the COVID-19 pandemic is reshaping the education service. In J. Lee \& S. H. Han (Eds.), The Future of Service Post-COVID-19 Pandemic (pp. 15-36). Springer. https:// doi.org/10.1007/978-981-33-4126-5_2

König, J., Jäger-Biela, D. J., y Glutsch, N. (2020). Adapting to online teaching during COVID-19 school closure: teacher education and teacher competence effects among early career teachers in Germany. European Journal of Teacher Education, 43(4), 608-622. https://doi.org/10.1080/02619768.2020.1809650

Lee, Y. C., Malcein, L. A., y Kim, S. C. (2021). Information and Communications Technology (ICT) Usage during COVID-19: Motivating Factors and Implications. International journal of environmental research and public health, 18(7), 3571. https://doi. org/10.3390/ijerph18073571

Lorduy, D., y Naranjo, C. (2020). Tecnologías de la información y la comunicación aplicadas a la educación en ciencias. Praxis $\mathcal{E}$ Saber, 11(27), e11177. https://doi.org/10.19053/22160159. v11.n27.2020.11177

Lorente, L. M. L., Arrabal, A. A., y Pulido-Montes, C. (2020). The Right to Education and ICT during COVID-19: An International Perspective. Sustainability, 12(21), 9091. https://doi. org/10.3390/su12219091

Lozano-Díaz, A., Fernández-Prados, J. S., Figueredo Canosa, V., y Martínez Martínez, A. M. (2020). Impactos del confinamiento por el COVID-19 entre universitarios: Satisfacción Vital, Re- siliencia y Capital Social Online. International Journal of Sociology of Education, 9(Extra 1), 79-104. http://doi.org/10.17583/ rise. 2020.5925

Menéndez Otero, C. (2020). “¡No es esto, no es esto!”: reflexiones docentes desde una universidad presencial (presuntamente) virtualizada. Magister, 32(1), 129-136. https://doi.org/10.17811/ msg.32.1.2020.129-136

Naresh, R. (2020). Education after COVID-19 Crisis Based on ICT Tools. UGC CARE Journal, 31(37), 464-468. https://doi. org/10.1186/s41239-0170070-1

Paudel, P. (2021). Online education: Benefits, challenges and strategies during and after COVID-19 in higher education. International Journal on Studies in Education (IJonSE), 3(2), 70-85. https://doi.org/10.46328/ijonse.32

Pozo-Sánchez, S., López-Belmonte, J. Fuentes-Cabrera, A., y López-Núñez, J. A. (2021). Aplicación trietápica del flipped learning en el área de las ciencias. Campus Virtuales, 10(1), 3547. http://www.uajournals.com/ojs/index.php/campusvirtuales/article/view/714/428

Rahiem, M. D. (2020). Technological barriers and challenges in the use of ICT during the COVID-19 emergency remote learning. Universal Journal of Educational Research, 8(11B), 61246133. http://doi.org/10.13189/ujer.2020.082248

Reinoso, R., Delgado-Iglesias, J., y Fernández, I. (2021). Effect of flipped teaching on the performance and perceptions of pre-service teachers on a biology course. Information and Learning Sciences, 122(1/2), 82-102. https://doi.org/10.1108/ILS-072020-0173

Rodicio-García, M. L., Ríos-de-Deus, M. P., Mosquera-González, M. J., y Penado Abilleira, M. (2020). La Brecha Digital en Estudiantes Españoles ante la Crisis de la Covid-19. Revista Internacional De Educación Para La Justicia Social, 9(3), 103-125. https://doi.org/10.15366/riejs2020.9.3.006

Rueda-Gómez, K. L. (2020). Estrategia educativa remota en tiempos de pandemia. Magister, 32(1), 93-96. https://doi. org/10.17811/msg.32.1.2020.93-96

Sandoval, C. H. (2020). La Educación en Tiempo del Covid-19 Herramientas TIC: El Nuevo Rol Docente en el Fortalecimiento del Proceso Enseñanza Aprendizaje de las Prácticas Educativa Innovadoras. Revista Tecnológica-Educativa Docentes 2.0, 9(2), 24-31. https://doi.org/10.37843/rted.v9i2.138

Shehzadi, S., Nisar, Q. A., Hussain, M. S., Basheer, M. F., Hameed, W. U., y Chaudhry, N. I. (2020). The role of digital learning toward students' satisfaction and university brand image at educational institutes of Pakistan: a post-effect of COVID-19. Asian Education and Development Studies, 10(2), 276-294. https:// doi.org/10.1108/AEDS-04-2020-0063

Tirado, S., Vázquez, A., y Toledano, R. (2021). La Docencia Virtual o e-Learning como Solución a la Enseñanza de la Física y Química de los Futuros Maestros en tiempos de COVID-19. Revista Española de Educación Comparada, 38, 190-210. https:// doi.org/10.5944/reec.38.2021.28853

Torralba-Burrial, A. (2020). Afrontando con el alumnado la evaluación online de una didáctica del medio natural en tiempos de la COVID-19. Magister, 32(1), 111-121. https://doi. org/10.17811/msg.32.1.2020.111-121

Van Vaerenbergh, S. (2020). Adaptación rápida a un modelo de docencia virtual: estudio de caso en la asignatura Didáctica de la Geometría. Magister, 32(1), 66-71. https://doi.org/10.17811/ msg.32.1.2020.66-71

Vélez-Loor, M. D., Vallejo-Valdivieso, P. A., y Moya-Martínez, M. E. (2020). Virtual teaching resources in natural science projects during confinement period by COVID-19. EPISTEME KOINONIA-Revista Electrónica de Ciencias de la Educación, Humanidades, Artes y Bellas Artes, 3(5), 183-201. http://dx.doi.org/10.35381/e.k.v3i5.760

Villalustre, L., Del Moral, M.E., y Neira-Piñeiro, M. R. (2019). Percepción docente sobrela realidad aumentada en la enseñan- 
za de Ciencias en Primaria. Análisis DAFO. Revista Eureka sobre Enseñanza y Divulgación de las Ciencias, 16(3), 3201. http:// doi.org/10.25267/Rev_Eureka_ensen_divulg_cienc.2019.v16. i3.3301
Wind, T. R., Rijkeboer, M., Andersson, G., y Riper, H. (2020). The COVID-19 pandemic: The 'black swan'for mental health care and a turning point for e-health. Internet interventions, 20, 100317, 1-2. http://doi.org/10.1016/j.invent.2020.100317

\section{Anexo 1}

Tabla 1. Enunciados de las cuatro preguntas realizadas a los estudiantes

\begin{tabular}{|c|l|}
\hline $\begin{array}{c}\text { Número } \\
\text { de pregunta }\end{array}$ & \multicolumn{1}{c|}{ Enunciado } \\
\hline 1 & $\begin{array}{l}\text { Después de trabajar durante las últimas semanas de forma online mediante el uso de diferentes estrategias } \\
\text { y recursos, ien qué grado consideras que esta forma de trabajar mejora tu aprendizaje en didáctica de las } \\
\text { ciencias respecto a la presencial? }\end{array}$ \\
\hline \multirow{2}{*}{2} & $\begin{array}{l}\text { Valora del 1 (nada) al 5 (mucho), el grado que cada una de las siguientes actividades y estrategias utilizadas } \\
\text { en clase, han contribuido a la mejora de tu aprendizaje en didáctica de las ciencias. } \\
\text { a) Videoconferencias o videoclases expositivas por parte del profesor } \\
\text { b) Videoclases interactivas con preguntas síncronas con Mentimeter } \\
\text { c) Visualización de forma individual de videos de YouTube con preguntas en Edpuzzle } \\
\text { d) Trabajo en grupo en salas alternativas de Google meet } \\
\text { e) Formularios en línea con Google Forms } \\
\text { f) Cuestionarios en línea con Kahoot y Quizizz } \\
\text { g) Realización de glosarios y repositorios de forma colaborativa }\end{array}$ \\
\hline h) Prácticas de ciencias experimentales mediadas con tecnología (la caja negra) \\
i) Indagación guiada (WebQuests) \\
j) Breakout edu (recapitulación de los contenidos trabajados)
\end{tabular}

